\title{
An On-the-Road Comparison of In-Vehicle Navigation Assistance Systems
}

\author{
David W. Eby and Lidia P. Kostyniuk, The University of Michigan Transportation \\ Research Institute, Ann Arbor, Michigan
}

\begin{abstract}
We compared system performance and driver opinion of 3 in-vehicle navigation aids - two advanced traveler information systems (ATISs; Ali-Scout and TetraStar) and written instructions - when used on the road concurrently under identical conditions. Few drivers in the study had difficulty finding initial routes or became lost. Users of Ali-Scout, an ATIS that utilizes traffic information in routing, drove longer-distance routes, got lost more frequently, and gave their system less positive ratings than did TetraStar users. Users of the 2 ATISs traversed routes that were significantly shorter in duration than those driven by users of written instructions. The time savings benefit of the advanced technology systems over written instructions was greatest during peak traffic conditions. Drivers who were familiar with the road network, overall, had less difficulty finding destinations and drove shorter-duration routes than drivers who were unfamiliar with the road network. Actual or potential applications of this research include improving the design of technologies that provide navigation assistance to travelers.
\end{abstract}

\section{INTRODUCTION}

One of the more interesting advances in transportation over the past decade is the introduction of intelligent transportation systems. Of particular interest is the flurry of activity directed at developing advanced traveler information systems (ATISs), especially systems that provide in-vehicle navigation assistance to drivers. These systems are designed to "acquire, analyze, communicate, and present information for use in assisting surface transportation travelers in moving from a starting location to their destination” (IVHS America, 1992, p. III-21). In other words, navigational ATISs give optimal route guidance information to destinations selected by the driver. As noted by several researchers (e.g., Eby, 1997; Fastenmeier, Haller, \& Lerner, 1994; Inman, Sanchez, Porter, \& Bernstein, 1995; IVHS America, 1992; Kantowitz, Kantowitz, \& Hanowski, 1995; Kostyniuk, Eby, Christoff, Hopp, \& Streff,
1997; Smiley, Vernet, Labiale, \& Pauzié, 1994; Srinivasan, Landau, \& Jovanis, 1995; Walker, Alicandri, Sedney, \& Roberts, 1990), the potential advantages of optimal routing are greater ease in finding destinations, fewer trips in which a driver gets lost, less complicated routes, avoidance of traffic congestion, shorter distance routes, shorter duration routes, greater confidence while driving, and less stressful driving. Collectively, these advantages potentially have the additional benefits of decreased fuel consumption and air pollution, less overall traffic congestion, and increased traffic safety. Understanding how people use ATISs and what they think about ATISs is of paramount importance in the development of systems that will provide these benefits.

Several in-vehicle navigation assistance systems have been developed in recent years. A basic difference among many of these systems is in their ability to use traffic conditions in determining the optimal route. Static route guidance 
systems determine the optimal route between some origin and destination (O-D) without taking into account traffic conditions that may be encountered during the trip. Systems that have the ability to use information about potential or actual traffic conditions to decide the optimal route are typically called dynamic route guidance systems. Because there is little agreement on the definition of dynamic route guidance (see, e.g., Eby, Kostyniuk, Christoff, Hopp, \& Streff, 1997; Schofer, Koppelman, Webster, Berka, \& Peng, 1996), we define the phrase broadly as route guidance that includes any traffic congestion information, including predictions of recurrent traffic congestion and real-time information that conveys both recurrent and nonrecurrent traffic congestion.

Further, some systems have information about the vehicle's location (e.g., through the satellite Global Positioning System [GPS] or dead reckoning) and can guide a driver by giving verbal and visual navigation instructions during the trip. Other systems show the entire route or set of navigation instructions to the driver in advance and give no additional guidance information during the trip.

Only a handful of studies have investigated the relative effectiveness of different navigational ATISs in providing some or all of the advantages discussed previously when tested under actual driving conditions (e.g., Eby, Kostyniuk, Christoff, et al., 1997; Fastenmeier et al., 1994; Inman et al., 1995; Parkes, Ashby, \& Fairclough, 1991; Schofer et al., 1996).

Schofer et al. (1996) studied the relative performance of an ATIS that was configured two different ways. In the first configuration, the ADVANCE system provided dynamic routing information while the driver drove to a specified destination that was based on both historic and real-time traffic information; that is, the configuration was able to use information about both recurrent and nonrecurrent traffic congestion information in route calculation. In the second configuration, routing was based only on historical, recurrent traffic congestion information. Although the system is dynamic according to the definition we use in this paper, Schofer et al. (1996) defined this configuration as static because the routing could not change once a trip began. In this study, pairs of drivers who were very familiar with the study road network started at the same origin and drove to the same destination at the same time. Drivers were required to follow the routing provided by the system, so the potential bias or superiority of a driver's own knowledge of routing would not influence test results. One driver's vehicle had the ADVANCE system in the first configuration and the other driver's vehicle was configured in the second way. The dependent variable in this study was trip time, and all 73 trials were run during peak traffic conditions. Schofer et al. (1996) found no consistent differences in travel time between the two configurations studied.

Although the Schofer et al. (1996) study provides much-needed information about ATIS performance (which was the intent of the study), it is limited in its analysis of potential benefits and in what it reveals about how drivers use ATIS. All data were collected from a small set of drivers who were familiar with the area and who drove only in peak traffic conditions, and only trip time was investigated. A more comprehensive study was conducted by Inman et al. (1995). In this study, three navigation assistance systems were investigated. The first system, Navigation Plus, presented dynamic navigation assistance to drivers as they drove, using routing that considered both recurrent and nonrecurrent congestion. The second system, Navigation, was the same as Navigation Plus except that its routing was static in that it used only road classification and no traffic information. The third "system" - map and instructions - used a paper map and writing utensils. Those using this system could call a human travel assistant prior to beginning their trip and get turn-by-turn instructions over the phone, which they could write down, or they could determine their own routing.

As in the Schofer et al. (1996) study, drivers drove between the same O-D pairs at the same time, with one driver using each type of system. Most participants were unfamiliar with the study area. All trials were run during peak traffic conditions, and a researcher rode along with drivers while they participated in the experiment. There were numerous dependent variables in the study, including trip time, trip distance, level of congestion, number of 
wrong turns, and crashes/near crashes. Several driver opinion measures were also collected, but only for drivers who used the Navigation and Navigation Plus systems.

Inman et al. (1995) found that drivers using Navigation Plus and Navigation had consistently shorter trip durations than did those using the map and written instructions. The addition of traffic information in routing (Navigation Plus) did not significantly reduce travel times as compared with the static Navigation system. Analysis of trip distance showed that drivers using Navigation traversed routes that were generally shorter than those who used either of the other two systems. This result implies that the map and instruction users probably did not use efficient routing in determining routes, whereas the Navigation Plus system may have routed people around traffic problems, leading to longer distance but shorter duration routes. Surprisingly, in the most general conditions of the study, no difference in levels of congestion encountered was found among users of the three systems. Analysis of wrong turns showed that roughly $40 \%$ of drivers made at least one wrong turn regardless of the system they were using. In their analysis of traffic safety, Inman et al. found that none of the 222 drivers were involved in a crash and only 16 were involved in near crashes, the highest percentage of which were in the written instructions condition.

The Inman et al. (1995) study effectively demonstrated the relative advantages of three distinct systems. However, this study, as well as the Schofer et al. (1996) study, was restricted to peak traffic conditions. Potential users of navigation assistance systems, particularly older people or people unfamiliar with the area, would typically drive during nonpeak traffic times (see, e.g., Eby \& Kostyniuk, 1998; Eby, Trombley, Molnar, \& Shope, 1998; Kostyniuk et al., 1997; Molnar, Eby, \& Hopp, 1996). Use of ATISs and the potential advantages of the system may differ widely depending on the traffic conditions in which the system is used. Further, both studies, either explicitly (Schofer et al., 1996) or implicitly (Inman et al., 1995), required the driver to follow the instructions they were given. Consequently, drivers' own routing abilities could not be utilized in conjunction with a system's routing abilities, nor could drivers deviate if they saw a potential traffic condition developing that they wanted to avoid, such as a crash or an emergency vehicle. Studies testing the use of ATISs in natural settings have shown that both situations are important to drivers (e.g., Chu, 1994; Eby, Kostyniuk, Streff, \& Hopp, 1997; Kostyniuk et al., 1997). Excluding these types of ATIS use may reflect advantages that do not apply when the systems are utilized in actual everyday driving activities.

The purpose of the present investigation was to compare system performance and driver opinion of two ATISs (TetraStar and AliScout) and written instructions when the systems were used concurrently under identical road conditions. As in the Inman et al. (1995) and Schofer et al. (1996) studies, identical conditions were achieved by having a trio of people drive similar vehicles at the same time to the same destinations. One person in the triplet used each system. So that we could generalize our results to a large set of potential ATIS users, drivers both familiar and unfamiliar with the study area participated during both peak and nonpeak traffic conditions. In addition, drivers were explicitly told that the routes given by the systems were only recommendations - they could opt to either follow these routes or use their own personal routing preferences.

\section{METHODS}

\section{Participants}

The 360 participants (51.9\% male) were paid for their participation. Their ages ranged from 19 to 80 years (mean $=37.4$ years). The number of participants was evenly divided among all study variables. The participants were recruited through advertisements in local and nonlocal newspapers. All respondents were asked their date of birth and whether they had a valid driver's license. Those not in possession of a valid license or who were under the age of 19 or over the age of 80 years were excluded from participation. Selfreported highest education levels showed that about $10 \%$ of the participants had a high school diploma or less, $47 \%$ had attended some college, and about $53 \%$ had a college 
degree or higher. Self-reported household income showed that about $38 \%$ of participants had a household income of less than \$25 000, about 38\% had a household income between \$35 000 and \$54 999, about 16\% had a household income between $\$ 55000$ and $\$ 79$ 999, and about $8 \%$ had a household income of $\$ 80000$ or greater.

More than one-half of study participants $(57 \%)$ indicated that when they drove in urban areas they generally listened to traffic reports, and nearly all participants $(98 \%)$ indicated that they were willing to divert from a driving route that they normally used in order to avoid traffic congestion. Roughly one-half of participants reported that they were not confident in their ability to navigate in unfamiliar areas. When asked about their frequency of road map use, $13 \%$ of the participants reported "at least once a week," 23\% reported "one to three times per month," $39 \%$ reported "once every 2 to 6 months," $11 \%$ reported "once a year," and 1\% reported "less than once a year." Analyses of all demographic factors showed that participant characteristics were evenly distributed over the study's independent variables.

\section{Design}

The three independent variables in the study were driver familiarity with road network (familiar, unfamiliar), traffic conditions (peak, nonpeak), and in-vehicle navigation assistance system (Ali-Scout, TetraStar, and written instructions). Driver familiarity was determined by self-report on a questionnaire completed at the time of recruitment. The study area was described to the participants, and they were asked whether they had either lived or worked in this area, and whether they thought that they were very familiar, familiar, somewhat unfamiliar, unfamiliar, or very unfamiliar with the study area. Those who indicated they had either lived or worked in the study area or were either familiar or very familiar with the study area were designated as "familiar" participants and were scheduled to participate in the familiar condition of the study. The remaining participants were considered "unfamiliar" and were scheduled for participation in that condition of the study.
Traffic condition was varied by running the study at different times of day on normal weekdays. No trials were conducted on weekends or holidays. Peak traffic conditions were defined as 7:00 a.m. to 9:00 a.m. and 4:00 p.m. to $6: 00$ p.m. Nonpeak traffic conditions were defined as 9:00 a.m. to 11:30 a.m. and 1:00 p.m. to 3:00 p.m.

Three in-vehicle navigation assistance systems were selected because of the different types of information they used and the different ways in which navigation assistance was provided. The Ali-Scout system was designed to determine the fastest route between the vehicle's current position and a user-supplied destination. Ali-Scout determined the fastest route by using road classification information combined with information about recurrent traffic congestion. Nonrecurrent traffic congestion, or real-time traffic information, was not used by this system. Route information and travel times along road segments (links) were transmitted between the vehicle and a network of roadside beacons with an infrared signal. Routes were calculated and link travel times were compiled on a central computer located at a traffic operations center run by the local road commission. Ali-Scout determined the vehicle's location through a deadreckoning calculation between roadside beacons and provided turn-by-turn instructions to a driver as he or she drove, using both visual and voice commands.

For every trip taken with the Ali-Scout system, two conceptually distinct kinds of guidance were used. At the start of a trip, Ali-Scout guidance began in autonomous mode. In this mode an arrow and number display showed only realtime distance and direction-to-the-destination information (i.e., "as the crow flies" information) without any turns being recommended. As the driver proceeded toward his or her destination, he or she eventually passed a roadside beacon, where a communication took place and a calculated route was downloaded by Ali-Scout. The system then changed to guided mode, in which the driver was given turn-by-turn instructions as he or she drove. An illustration of the Ali-Scout system showing a driving maneuver arrow and other navigation information is shown in Figure 1. When the vehicle was within 


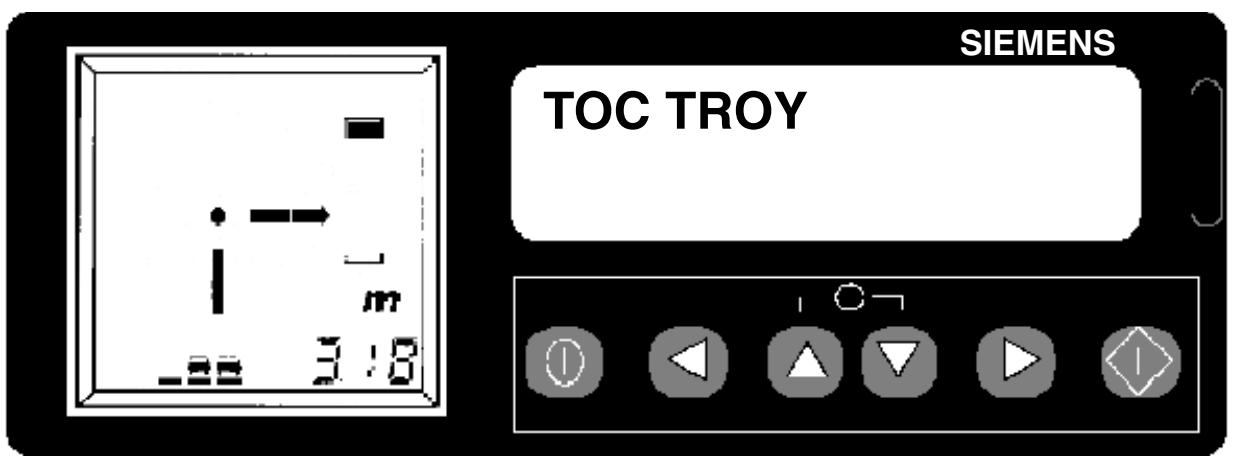

Figure 1. Illustration of an Ali-Scout unit showing a right-turn maneuver icon, recommended lanes for turn, distance (in miles), and countdown bar showing relative distance to the maneuver.

about 0.5 mile $(0.8 \mathrm{~km})$ of the destination, AliScout reverted back to autonomous mode guidance and the driver was required to look for the exact destination. Ali-Scout also reverted to autonomous mode guidance if the driver did not make a recommended maneuver or if communication at a beacon was disrupted (e.g., the beacon was not functioning or the infrared signal was blocked). When this occurred, Ali-Scout remained in autonomous mode until another beacon was passed.

The TetraStar in-vehicle navigation assistance system was similar to other commercially available products. TetraStar provided static route guidance only; that is, it determined the fastest route between the vehicle's current position and a user-supplied destination without using any information about traffic conditions. TetraStar determined the vehicle's location using an on-board global positioning system (GPS) and provided visual and voice, turn-byturn navigation assistance to the driver. The visual guidance instructions consisted of an electronic map, on which a highlighted route to the destination and the vehicle's current location were shown, and driving-maneuver icons. As a trip started, TetraStar displayed the map and both verbally and visually instructed the driver to proceed to the highlighted route, usually a few hundred meters from the vehicle's current location.

Once on the route, TetraStar began displaying turn-by-turn instructions by showing the next required maneuver, its distance away, and the name of the street where the maneuver was to occur. Figure 2 depicts the TetraStar system showing a driving maneuver icon and the other navigation information. Once the destination was within a few hundred yards, TetraStar reverted to the map display showing the highlighted route to the destination. If a driver failed to make a recommended turn, TetraStar stopped giving navigation instructions and prompted the user to press a button to recalculate the route.

The written instructions in-vehicle navigation assistance "system" represented a type of navigation assistance that most people have used, and it is the system that electronic systems must outperform if they are to be considered an improvement over the current technology. Unlike the Inman et al. (1995) study, in the present study, the written instructions were turnby-turn directions typewritten onto an $8.5 \times$ 11 -inch $(21.6 \times 29.9-\mathrm{cm})$ sheet of paper and were accompanied by a black-and-white map depicting the study area, with an origin and destination (O-D) indicated. In the Inman et al. study, only those drivers who wanted directions from an external source received them. In the present study, written instructions were given to everybody using this system so that all drivers, regardless of system, would have external routing recommendations for their trip if they wanted to use them. So that no a priori knowledge about traffic conditions on certain roadways was used in route selection, the routes between the O-D pairs used in the study were determined randomly from a set of equally direct routes. 


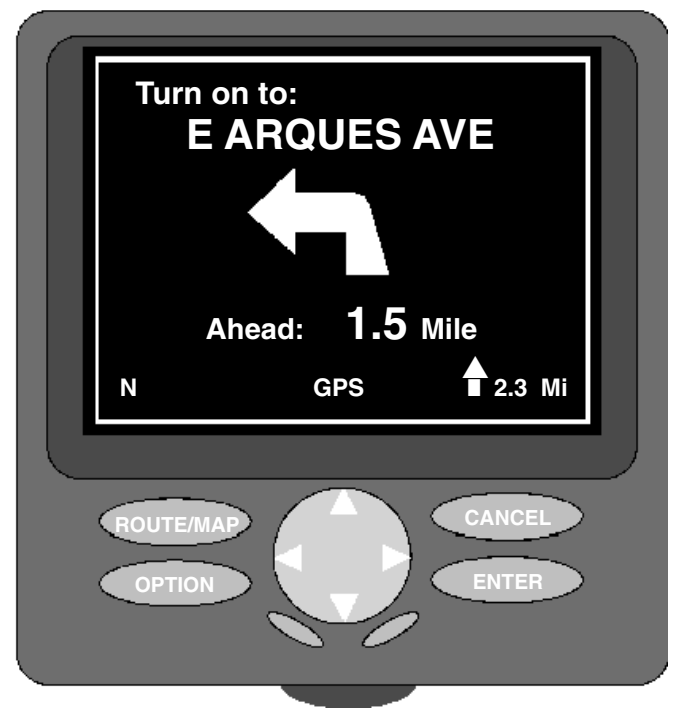

Figure 2. Illustration of a TetraStar unit showing a left-turn maneuver icon, the name of the street where the turn will take place, the distance to the maneuver (in miles), the compass direction the vehicle is traveling ( $\mathrm{N}=$ north), the status of the GPS system, and the distance (in miles) and direction to the destination.

The combination of independent variables in this study (system, familiarity, and traffic conditions) yielded 12 unique conditions - 30 drivers participated in each of these conditions. System and traffic condition were randomly assigned to a participant, and familiarity was based on a survey response as described earlier.

\section{Procedure}

The study was conducted during May, June, and July 1996 by a team of four research assistants housed in a field office located in the study area. An experimental session proceeded as follows. Sessions were run in sets of three participants. If three participants were not available, the session was not conducted. Upon arriving at the field office, the three participants were taken to a conference room, assigned randomly to a navigation system condition, and given a brief orientation on the administrative and procedural aspects of the study. All participants were told that their task would be to drive between a given origin and destination "as quickly and as safely as possible." They were told not to break any traffic laws and that they could take any route they chose but would be provided with a recommended route. The participants were informed that they would be driving two trips. The first trip was intended exclusively to provide the participants experience in using the navigation system and to familiarize them with the operation of the test vehicle. Participants were instructed that at the end of the first trip (the practice trip) they would be met by an experimenter, who would give them a new O-D pair to drive (the experimental trip). Participants were not told about the second destination until the first destination was reached, and they also were not told about the difference between the trips.

Prior to beginning the trips, the three participants were separated and given training that was specific to the navigation assistance system to which they were assigned. For the Ali-Scout and TetraStar participants, function and presentation of navigation assistance information was conveyed through the use of a model car, a schematic road network, and a series of printed graphics. Because the participant assigned to the written instructions condition needed no training, he or she waited in a reception area during this process.

After training, participants were brought to the test vehicles containing their assigned system, shown the navigation assistance system, and given a paper map that showed the O-D 
pair for the practice trip. At this time the writteninstructions participant was given the printed instructions. The Ali-Scout and TetraStar systems were already programmed with the proper destination by the researcher. In order to prevent participants from inadvertently turning off or reprogramming the Ali-Scout or TetraStar unit, those participants using these devices were instructed not to touch the device when driving.

Three O-D pairs were used in the study, with a single pair (randomly selected from the set of three) used each day. Each O-D pair was matched as closely as possible on the following variables: distance (about $11.7 \mathrm{~km}$ ), road classification, traffic control device use, land use, and type of traffic conditions during peak and nonpeak times. A complete description of the O-D pair matching procedures can be found in Eby, Kostyniuk, Christoff, et al. (1997).

When the participant was familiar with the vehicle and navigation system, he or she began driving. The experimenter ensured that there was at least a 5-min delay between the three drivers' departures to prevent them from following one another to the destination. After the practice trip, the driver was met at the first destination by an experimenter who start- ed the data collection equipment, entered the next destination (or handed out the next set of written instructions), showed the driver where the destination for the experimental trip was located on a paper map, and then let the participant begin driving. Again, a 5-min delay minimum was maintained between drivers.

At the completion of the experimental trip, another experimenter recorded data, shut off the data collection equipment, and gave the driver a brief questionnaire. When finished, the driver was paid and given a debriefing sheet that described the experiment in which he or she had just participated.

\section{Data Collection}

Automated. Each vehicle was equipped with a laptop computer placed on the floor in the back seating area of the vehicle. A Trimble Navigation Ltd. (Sunnyvale, CA) GPS system was plugged into the PCMCIA slot of the computer. Also attached to each computer through a serial port was a Differential Corrections Inc. (DCI; San Jose, CA) differential corrections receiver. The DCI system received differential corrections through an FM subcarrier signal broadcast in the study area. A schematic of the various components is shown in Figure 3.

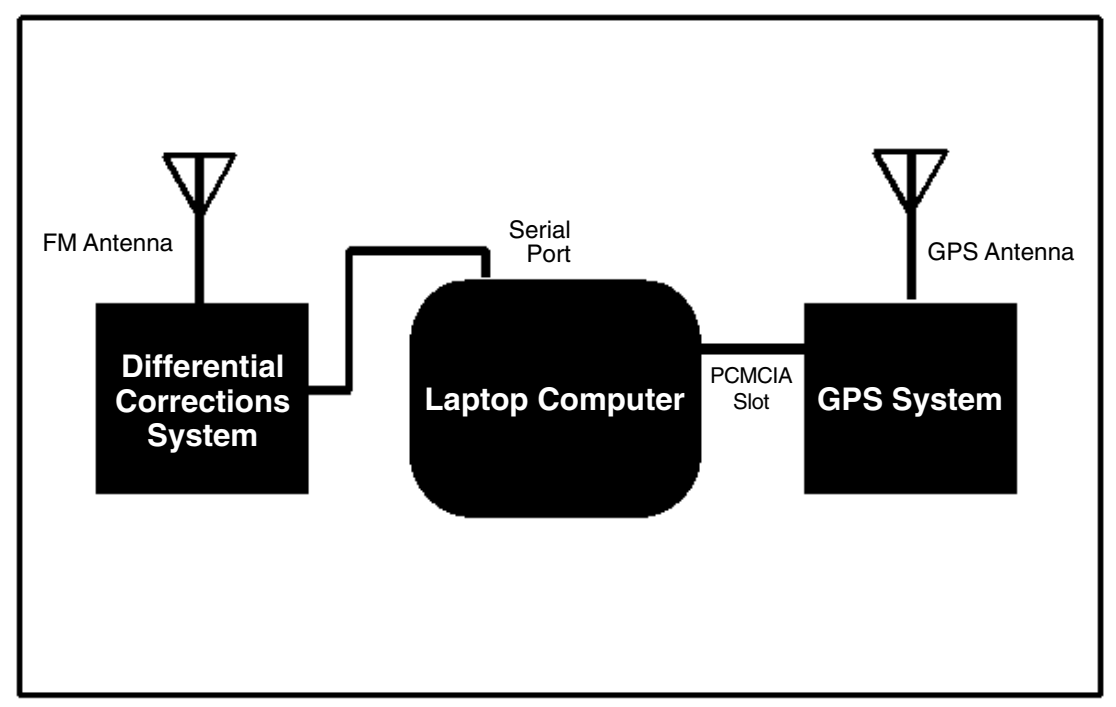

Figure 3. Diagram showing the automated data collection instruments and how they were related. 
The GPS information and differential corrections were integrated with Trimble software and read into a mapping program designed to work with the Trimble GPS hardware. The mapping program allowed us to track and record the exact time, position (latitude and longitude), speed, and heading of the entire trip on a second-by-second basis. We called these trip-records GPS logs. The following measures were derived from the automated data collection instruments:

1. Problem finding initial route? This was a yes/no measure. If the driver left the origin and immediately turned in the wrong direction, this was designated as having difficulty finding the initial route. This measure was included because both Ali-Scout and TetraStar require the driver to first find a route before turn-by-turn instructions are given. This measure is distinct from getting lost because a driver may have difficulty finding the initial route but then may drive directly to the destination. In this case, the trip was defined as one in which there was difficulty finding the initial route but was not defined as one in which the participant got lost.

2. Problem finding destination? This was a yes/no measure. If drivers were near the destination but either passed it or turned around before reaching it, they were designated as having difficulty finding the destination. For all three invehicle navigation assistance conditions, the drivers were given directions to the destination but were required to spot the exact destination for themselves. As in the previous measure, this measure was distinct from getting lost because a driver may drive directly to the destination but then have difficulty finding it. In this case, the trip was defined as one in which there was difficulty finding the initial destination but was not defined as one in which the participant got lost.

3. Did the participant get lost? This was a yes/no measure. Trips in which drivers made consistent maneuvers that took them away from the destination were recorded as those in which the participant got lost. Trips in which a vehicle remained stationary for more than 3 min were also recorded as lost because it was assumed that the driver was receiving additional navigation assistance by asking directions, consulting a street map, or calling an experimenter.

4. Number of turns. As a way of measuring the complexity of the route taken, the number of turns was analyzed. This measure was a tally of all turns taken by the driver and did not include turns taken in the parking areas of the origins and destinations. U-turns were counted as a single turn.
5. Number of navigation errors. This measure was a tally of all navigation errors made by the driver. A navigation error was defined as a turn that took the driver away from the destination or a failure of the driver to make a turn that should have been made, such as turning into a destination parking area.

6. Time spent at zero velocity. This measure was the total number of seconds during a trip in which the vehicle remained stationary. This dependent variable was included as a way of measuring the amount of congestion encountered during a trip.

7. Trip distance. This measure was the exact distance for the trip.

8. Trip duration. This measure was the time between when the vehicle began to move at the origin and the point where it stopped at the destination.

Because we monitored each automated data collection system for proper functioning several times a day, two complete systems were available for backup, and a backup system could be swapped with a malfunctioning system in a few minutes, we experienced data collection problems on only $5.4 \%$ of trips. For these trips, data for the first six measures described could not be obtained. As a backup precaution, trip distance was obtained from the in-vehicle odometer, which was reset by the experimenter prior to each trip, and trip duration was collected from a stopwatch left in the vehicle and operated by the researchers.

Questionnaire. At the end of the study, all drivers were asked to complete a questionnaire. The questionnaire asked the drivers about how they used their navigation assistance system and what they thought about it. The questionnaire required about $10 \mathrm{~min}$ to complete.

Drivers were asked about the frequency with which they followed the navigation system turn recommendations, their level of satisfaction with the navigation system they used, their level of distraction while using the system, their level of confidence in the accuracy of the system, how helpful the system was in finding destinations, and how safe they felt while using the system. Drivers responded to questions by indicating their answers on 7point scales that were anchored with appropriate labels. The question about frequency was anchored with the label never for 1 and 
always for 7. Questions about level were anchored with the labels very [item] for 1 (e.g., very satisfied) and not at all [item] for 7 (e.g., not at all satisfied). The question about perceived safety had a scale anchored with the labels much more safe for 1 and much less safe for 7 .

\section{RESULTS}

Although 30 drivers participated in each cell of the experiment, because of failures in data collection equipment and participants declining or giving invalid answers on survey items, the number of cases on some measures is slightly less than 30 per cell.

Automated data were analyzed using analysis of variance (ANOVA), Fisher's exact tests (Mehta \& Patel, 1983), or chi-square tests, depending upon the type of data. The ANOVA was used to test for differences among means within and between independent variables (invehicle navigation system, driver familiarity, and traffic conditions) for the following dependent variables: time spent at zero velocity, trip distance, trip duration, and the subjective ratings collected from the questionnaire. Two-tailed Fisher's exact tests were used to determine if there was an association among levels of the independent study variables for four dependent variables: finding the initial route, finding the destination, getting lost, and number of navigational errors. Chi-square tests were used to determine if there was an association among levels of the independent study variables for the number-of-turns dependent variable. Statistical significance is reported for all tests at a probability equal to or less than .05.

\section{Automated Data}

Finding the initial route. Overall, only $1 \%$ of drivers had difficulty finding the starting point of the route. All who had this difficulty were using the Ali-Scout system, all were unfamiliar with the road network, and onehalf drove during peak traffic periods. A twotailed Fisher's exact test showed that there was a statistical difference in ability to find the initial route among systems $(p=.036)$. Because difficulty finding the route was found only with users of Ali-Scout, this result occurred because of the poorer performance of AliScout on this measure. All other comparisons were nonsignificant.

Finding the destination. Overall, 3\% of drivers had difficulty locating the destination once they were near it. Of these, $78 \%$ were using the Ali-Scout system, $11 \%$ were using the TetraStar system, and $11 \%$ were using written instructions. About one-half of these drivers were unfamiliar with the area, and about one-half drove in nonpeak traffic. A two-tailed Fisher's exact test revealed a reliable difference among systems on the difficulty of finding the destination $(p=.030)$. The fact that the vast majority of the drivers who had difficulty finding the destination were using Ali-Scout suggests that the effect of system found here occurred because of the poorer performance of Ali-Scout as compared with the other two systems. There was no statistical difference found for driver familiarity or traffic conditions.

Getting lost. Overall, drivers got lost on only $2 \%$ of the trips. Of these participants, $86 \%$ were using Ali-Scout and 14\% were using written instructions. No drivers got lost using the TetraStar system. The drivers who got lost were about equally distributed by level of familiarity, and $86 \%$ of these trips were in nonpeak traffic. A two-tailed Fisher's exact test showed a statistically significant difference between systems $(p=.019)$ for this measure. Again, the fact that nearly all drivers who got lost were using Ali-Scout suggests that the statistical difference occurred because of the poorer performance of Ali-Scout users as compared with users of the other two systems. There were no significant differences for the other independent variables. Because of the significant difference among systems on getting lost, and because getting lost affects the number of turns, number of navigation errors, time spent at zero velocity, trip length, and trip duration, the analyses of the remaining automatically collected data exclude trips in which the driver got lost.

Number of turns. To determine if the system used, the driver's familiarity with the area, or the traffic condition influenced the complexity of the trip, a route complexity measure 


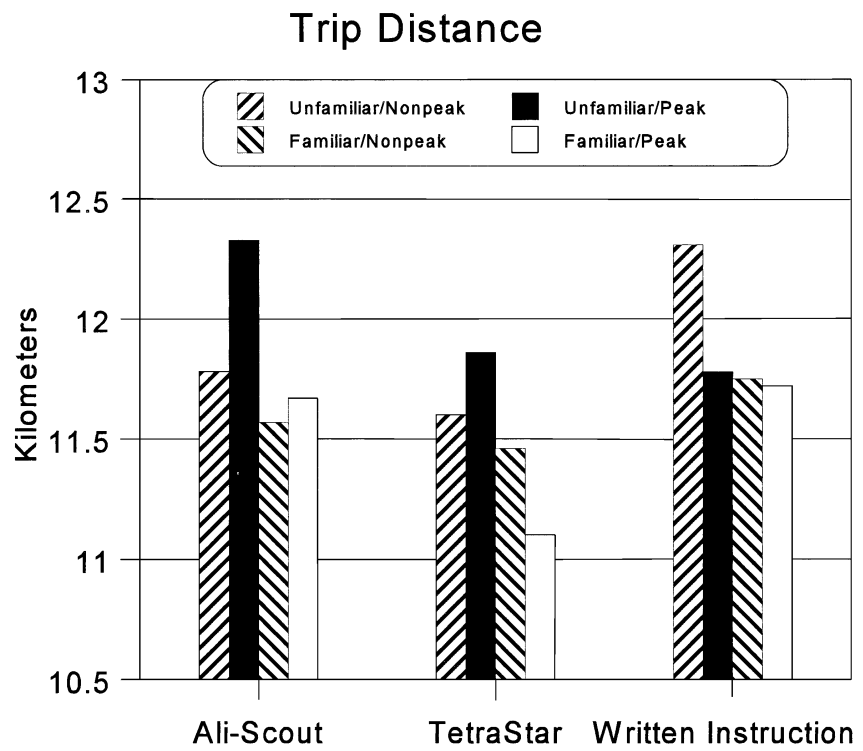

Figure 4. Average trip distance in kilometers as a function of system, driver familiarity, and traffic condition. Trips in which the driver got lost are not included.

based on the number of turns above the minimum needed to drive from the origin to the destination (three) was defined. Trip complexity was categorized as taking zero, one, two, or three or more turns over the minimum needed to drive from the origin to the destination. The zero, one, and three or more extra turns categories each had about $20 \%$ of the drivers, and about $40 \%$ of drivers were in the two extra turns category. Of Ali-Scout users, 44\% made zero extra turns, $40 \%$ made one, $29 \%$ made two, and $24 \%$ made three or more. Of TetraStar users, 30\% made zero extra turns, 39\% made one, $41 \%$ made two, and $18 \%$ made three or more. Of the users of written instructions, $15 \%$ made zero extra turns, $12 \%$ made one, $45 \%$ made two, and $27 \%$ made three or more. The distributions of route complexity for both levels of driver familiarity and types of traffic conditions were similar to the overall distribution.

Chi-square tests revealed that there was a significant difference among navigation assistance systems on route complexity, $\chi^{2}(6)=13.63, p=$ .034. There was no statistical difference by familiarity, $\chi^{2}(3)=0.333, p=.95$, or traffic condition, $\chi^{2}(3)=2.631, p=.452$. The effect of route complexity and navigation system was further examined by pairwise comparisons of the navigation systems. This analysis showed that the main effect of system resulted from the statistical difference between Ali-Scout and written instructions, $\chi^{2}(3)=11.892, p=.008$. All other comparisons were nonsignificant.

Number of navigational errors. Most of the drivers who did not get lost also made no navigational errors. Of those who got lost, 5\% made one navigational error and $1 \%$ made two such errors. Separate two-tailed Fisher's exact tests showed that the number of navigational errors did not vary significantly as a function of any of the independent variables in this study.

Time spent at zero velocity. The average time during a trip in which drivers were not moving was 164 s. As expected, a 3 (navigation assistance system) $\times 2$ (familiarity) $\times 2$ (traffic condition) between-subjects ANOVA (three-way) revealed a highly significant main effect of traffic conditions, $F(1,323)=15.98, p<.0001$. Regardless of the system used or driver familiarity, drivers in peak traffic conditions spent more time waiting $(189 \mathrm{~s})$ than did drivers who drove during nonpeak times (145 s). Time spent at zero velocity did not consistently differ among navigation systems or level of driver familiarity.

Trip distance. The trip distance averaged over all study variables was $11.7 \mathrm{~km}$. The average trip distance as a function of system, traffic conditions, and driver familiarity is shown in 


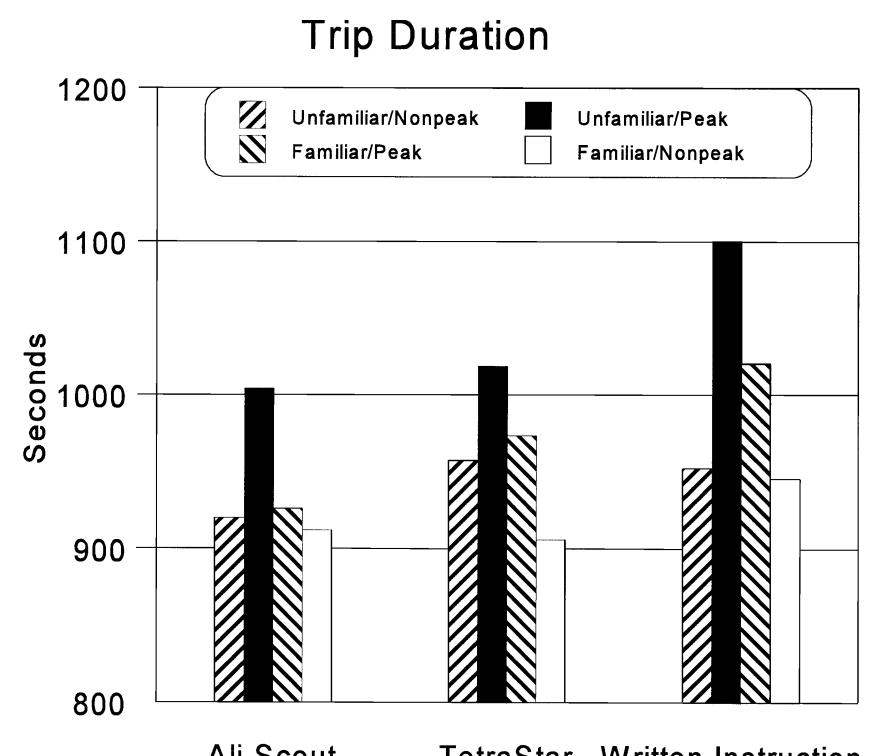

Figure 5. Average trip duration in seconds as a function of system, driver familiarity, and traffic condition. Trips in which the driver got lost are not included.

Figure 4. A three-way ANOVA revealed a significant main effect of navigation system, $F(2,340)=3.21, p<.05$. Post hoc analyses showed that this effect resulted from the fact that drivers using TetraStar consistently drove shorter routes (11.4 $\mathrm{km}$ on average) than drivers using either Ali-Scout (11.9 km) or written instructions $(11.9 \mathrm{~km})$. There was also a significant main effect of driver familiarity, $F(1,340)=$ 8.52, $p<.01$. Drivers who were familiar with the road network utilized in the study drove routes that were consistently shorter $(11.6 \mathrm{~km}$ on average) than those driven by unfamiliar drivers $(11.9 \mathrm{~km})$. All other main effects and interactions were nonsignificant.

Trip duration. Averaging across all trips (except those in which the driver got lost, which were removed from the analysis), the average trip duration was $970 \mathrm{~s}$, or about 16 min. The average trip duration by traffic conditions, driver familiarity, and system is shown in Figure 5. A three-way ANOVA showed significant main effects of traffic conditions, $F(1,340)=23.34, p<.0001$, driver familiarity, $F(1,340)=8.36, p<.005$, and system, $F(2,340)=5.77, p<.005$. All interactions were nonsignificant. As expected, drivers participating in peak traffic conditions drove routes that were consistently longer in dura- tion (1007 s) than drivers who drove during nonpeak times (932 s). In addition, the effect of driver familiarity was attributable to the fact that familiar drivers drove routes that were of shorter duration (947 s) than the routes driven by unfamiliar drivers (992 s).

The effect of system occurred because users of both Ali-Scout (941 s) and TetraStar (964 s) had shorter duration trips than those using written instructions (1005 s). Post hoc comparisons between navigation systems showed that these differences were statistically significant, $F(1,224)=12.07, p<.001$, for Ali-Scout versus written instructions; and $F(1,230)=$ 5.24, $p<.05$, for TetraStar versus written instructions. There was no reliable difference between Ali-Scout and TetraStar on trip duration; each averaged about $50 \mathrm{~s}$ (about 5\%) faster than trips taken with written instructions. Under nonpeak traffic conditions, the time savings of the two ATISs over written instructions was $1.4 \%$ (13.5 s) for unfamiliar drivers and $3.8 \%$ (36.5 s) for familiar drivers; at peak traffic conditions the time savings grew to $9.3 \%(89.2 \mathrm{~s})$ for unfamiliar drivers and $7.3 \%$ (70.2 s) for familiar drivers. This $5 \%$ time savings averaged over multiple trips represents a potential significant reduction in the amount of time and fuel spent traveling. 


\section{Questionnaire Data}

All drivers were asked to complete a questionnaire, and none declined. However, some drivers declined to answer certain questions or gave invalid answers. Only valid answers were included in the questionnaire analyses.

Drivers were asked, "Considering both trips that you drove in this study, how often did you follow the recommendations to turn?" Overall, drivers reported that they nearly always followed the navigation recommendations they received. Across all conditions, $84.6 \%$ of respondents responded with either a 6 or 7 to this question. A three-way ANOVA revealed that there were no significant differences among respondents on any of the study variables.

Drivers were instructed to "Please indicate your level of satisfaction with the navigation assistance available to you while driving in the study." Responses showed that drivers were generally very satisfied with the system they used in the study. A three-way ANOVA showed that there were no significant differences in level of satisfaction for any of the study variables except for a highly reliable main effect of system, $F(2,333)=11.40, p<.0001$. Post hoc analyses showed that this difference resulted from the fact that Ali-Scout users were less satisfied overall than users of the other two systems. Averaging across the driver familiarity and traffic condition variables, we found that $63.8 \%$ of Ali-Scout users responded with either a 1 or 2 , whereas $82.9 \%$ of TetraStar users and $87.5 \%$ of written instruction users responded with either a 1 or 2.

Drivers were instructed to "Please indicate your level of distraction while using the navigation assistance available to you while driving in the study." Overall, drivers reported that the level of distraction while driving was generally low, with $76.6 \%$ of respondents indicating either a 6 or 7 . A three-way ANOVA showed that there were no significant effects for level of distraction except for a main effect of system, $F(2,339)=7.27, p<.001$. Post hoc analyses showed that this difference resulted from the fact that users generally reported higher levels of distraction for the Ali-Scout system than for TetraStar system or written instructions. On average, $62.7 \%$ of
Ali-Scout users reported at least some level of distraction using the system (i.e., a response of less than 7 ), whereas $48.7 \%$ of TetraStar users and $41.2 \%$ of written instruction users reported some level of distraction using the system.

Drivers were instructed to "Please indicate your level of confidence in the accuracy of the navigation assistance available to you while driving in the study." Overall, responses showed a high level of confidence in the accuracy of the navigation assistance systems. A three-way ANOVA showed that there were no significant differences in level of confidence in the navigation accuracy except for a main effect of system, $F(2,343)=9.45, p<.0005$. Post hoc analyses showed that this difference resulted from the fact that users reported less confidence in the accuracy of Ali-Scout navigation assistance than the assistance received with either TetraStar or written instructions. Averaging across driver familiarity and traffic conditions, we found that a high level of confidence (i.e., a response of either 1 or 2) was reported by $66.4 \%$ of Ali-Scout users, whereas $86.7 \%$ of TetraStar and $91.4 \%$ of written instruction users reported high levels of confidence in the navigation accuracy of the assistance received in the study.

Drivers were instructed to "Please indicate how helpful the navigation assistance you received during the study was for you in finding the study destinations." Averaged over all conditions in the study, $80.3 \%$ of drivers indicated high levels of helpfulness (i.e., a response of either 1 or 2). A three-way ANOVA showed that there were significant differences in level of helpfulness by system, $F(2,343)=$ $5.34, p<.01$, and driver familiarity, $F(1,343)=$ $9.51, p<.005$. Post hoc analyses revealed that the main effect of system resulted from the fact that users of written instructions reported higher levels of helpfulness in finding study destinations than did users of the other two systems. In addition, TetraStar users reported higher levels of helpfulness than did users of Ali-Scout. Averaging across familiarity and traffic conditions, $73.1 \%$ of users rated AliScout as providing high levels of helpfulness, $81.5 \%$ of TetraStar users ranked it as providing high levels of helpfulness, and $86.3 \%$ of 
the written instructions users ranked them as high in helpfulness. As expected, the effect of driver familiarity on reported helpfulness resulted from the fact that familiar drivers found the systems to be less helpful in finding destinations than did unfamiliar drivers. On average, $86.5 \%$ of unfamiliar users reported high levels of helpfulness, compared with only $74.0 \%$ of familiar drivers. No other main effects or interactions were significant.

Finally, drivers were instructed to "Please indicate how safe you felt driving while using the navigation assistance available to you in the study as compared with your everyday driving." Overall, $64.2 \%$ of drivers reported at least some increase in feelings of safety (i.e., a response of less than 4) over their normal driving. A three-way ANOVA revealed that there were no significant main effects of system, driver familiarity, or traffic conditions.

\section{DISCUSSION AND CONCLUSIONS}

The purpose of this study was to compare how drivers used and what they thought about Ali-Scout, TetraStar, and written instructions. The participant group was composed of drivers who were familiar and those who were unfamiliar with the road network in the study area, and the study was conducted during peak and nonpeak traffic times. The three systems were compared through observation of driving behaviors (as determined by a GPS tracking system) and through self-reported use and opinions.

Even though the drivers had the option of deviating from the routes recommended by their navigation system, most reported following the turn recommendations frequently regardless of the system they used. We found that few drivers had difficulty finding initial routes or got lost during the study. However, users of Ali-Scout had significantly more difficulty finding the route starting points and destinations than did drivers using either of the other systems. Thus, Ali-Scout, which had dynamic routing information, performed less well than the ATIS with static information on these measures.

The inclusion of traffic information in the route calculation should not affect either of these measures, so these results are most likely related to the method of presenting guidance information used by Ali-Scout at route start and end points. The Ali-Scout system presented only distance and direction-to-destination (autonomous) guidance information at route start and end points. It may be that users of an ATIS need turn-by-turn guidance that starts from when they begin driving and continues until they pull into their destination. This type of guidance was provided by both TetraStar and written instructions in the present study. Previous studies of Ali-Scout have also shown that people dislike autonomous guidance information and consider it less helpful for finding destinations than turn-by-turn instructions (Eby, Kostyniuk, Streff, et al., 1997; Kostyniuk et al., 1997).

The study also showed that few drivers got lost using any of the systems, including drivers who were unfamiliar with the area, suggesting that all three systems were good at providing navigation assistance. When the systems were compared on this variable, however, we found that the majority of lost drivers were using Ali-Scout. Again, because getting lost is not necessarily related to the inclusion of traffic information in route calculation, this result is more likely related to the unique characteristics of the Ali-Scout system. However, dynamic systems that have the ability to change routing based on new traffic information, such as AliScout or Navigation Plus, must have a means of communicating with a centralized facility. With Ali-Scout, this communication was through roadside beacons. If a beacon was not operational or the infrared communication signal was blocked by a truck or bus, then turnby-turn instructions would have been lost midroute or would never have started. Drivers depending on the Ali-Scout information may have become lost when this occurred. In fact, during debriefing, several drivers who got lost mentioned this as a contributing factor in their getting lost. Thus, it is possible that dynamic systems using a communication line (cell phone, FM radio, or beacons) may pose a higher risk for getting drivers lost.

The study showed that few drivers made navigational errors regardless of the system used, their familiarity with the area, or traffic 
conditions. These results differ from those of Inman et al. (1995), who showed that navigational errors were made on about $40 \%$ of trips. These results show that all systems, even the written instructions, were good at providing navigation assistance.

One potential benefit of dynamic over static guidance systems is that the former could help the driver to avoid traffic congestion. One measure of the amount of traffic congestion encountered during a trip is the amount of time spent at zero velocity (wait time). Overall, we found no difference for this variable among navigation systems, confirming the results of Inman et al. (1995). Thus, it appears that either the dynamic information used by AliScout was not accurate or there simply were no alternate routes with less congestion.

The analysis of trip distance showed that, overall, trips driven with Ali-Scout tended to be of longer distance than trips taken by users of TetraStar or written instructions; that is, users of the dynamic system (Ali-Scout) traversed routes that were of longer distance than the trips traversed by users of the other two systems. This finding is similar to the results of Inman et al. (1995). In their study, users of the dynamic Navigation Plus system and the static map and instructions system drove longer distance routes than users of the static Navigation system. The difference between the results obtained with the map and instructions condition in their study and those with the written instructions condition in the present study is most likely attributable to the fact that all drivers in this study had written instructions, whereas in the Inman et al. study only those drivers who wanted directions from an external source received them.

Perhaps the most important comparison of the systems in this study was the analysis of trip duration. As expected, for each system studied, we found that drivers who were familiar with the road network in the study area drove shorter duration trips than drivers who were unfamiliar with the network. Also as expected, trip durations were longer during peak traffic times than nonpeak times. The traffic condition variable is of particular interest because Ali-Scout was designed to provide routing that avoids high-traffic areas by access- ing traffic information when determining routing. The potential benefit of this feature should be greatest during peak traffic conditions. If Ali-Scout were to provide additional trip-time savings over the other static systems during peak traffic times, we would expect to find a significant interaction between navigation system and traffic condition. We did not find this interaction in our statistical comparisons.

Consistent with the results of Inman et al. (1995), we found that users of the two advanced technology systems drove routes that were nearly identical in duration and that these were significantly faster than trips taken by users of written instructions. Thus, when lost drivers are removed from the analysis, it appears that guidance instructions provided by in-vehicle electronic technology lead to routes that are faster than those provided by written instructions.

Because the study showed that users of written instructions drove routes that were either shorter or the same distance as users of the other two systems and the level of congestion encountered did not differ among systems, the longer trip durations for the written instructions must have been caused by drivers with the map and instructions driving more slowly than users of the other systems. One primary difference between in-vehicle technology and written instructions is that users of written instructions need to remember an entire set of route instructions (or at least several maneuvers) in order to get to a destination. Frequently, as in the present study, the number of maneuvers would exceed most drivers' short-term memory capacity (Miller, 1956). Therefore, drivers using the map and instructions in the present study may have had to pay some attention to rereading instructions or looking at the map while they were driving.

Research has shown that when drivers devote attention to a conversation on a cellular phone while driving, their speed decreases (e.g., Fairclough, Ashby, Ross, \& Parkes, 1991). A similar effect may have occurred with the written instructions used in this study.

The participants' opinions of the various systems showed consistent results. A large percentage of study participants were very satisfied with the system they used, reported at 
TABLE 1: Comparison of the Systems on Each Measure in the Study

\begin{tabular}{llcc}
\hline & & System & \\
\cline { 2 - 4 } Measure & Ali-Scout & TetraStar & Written \\
\hline Finding the initial route & Bronze & Gold & Gold \\
Finding the destination & Bronze & Gold & Gold \\
Preventing driver from getting lost & Bronze & Gold & Silver \\
Trip complexity & Gold & Gold & Bronze \\
Preventing navigational errors & Gold & Gold & Gold \\
Shortest time spent waiting & Gold & Gold & Gold \\
Shortest trip distance & Gold & Silver & Silver \\
Shortest trip duration & Gold & Gold & Bronze \\
Reported frequency of following & Gold & Gold & Gold \\
ATIS recommendations & & & Gold \\
Reported level of satisfaction with system & Bronze & Gold & Gold \\
Least reported distraction & Bronze & Gold & Gold \\
Reported confidence in system accuracy & Bronze & Silver & Gold \\
Reported level of helpfulness & Bronze & Silver & Gold \\
Reported level of driving safety & Gold & Gold &
\end{tabular}

least some level of distraction while driving, had very high levels of confidence in the accuracy of the system, thought the system was helpful in finding their destination, and experienced increased feelings of safety while using the system. Comparisons among systems on these variables showed that Ali-Scout users reported less satisfaction, higher levels of distraction while driving, and lower levels of helpfulness in finding destinations than did users of either TetraStar or written instructions. TetraStar and written instruction users did not differ greatly on any of these same variables. Overall, TetraStar and written instructions systems were more highly valued than Ali-Scout. This is not surprising, given that we also found that the dynamic feature of Ali-Scout did not seem to provide faster routes or routes that were less congested than those provided by TetraStar.

A summary of how the systems compared on each measure in the study is shown in Table 1. In this table we use the Olympic medal-color classification to rank the systems because for many of the measures, placing second or third was still good. For those measures in which there was no difference between systems, multiple medals of the same color were awarded. This table clearly shows that the TetraStar system received or was tied for the highest ranking (gold) on 11 of the 14 measures. Over all study measures, written instructions was ranked second, receiving or being tied for the highest ranking on 10 of the 14 measures. The thirdplace system was Ali-Scout, which received or was tied for the highest ranking on only onehalf of the measures.

Ali-Scout's poorer showing in comparison with the other two systems in this study probably results from two factors. First, drivers had some difficulty with the autonomous mode guidance ("as the crow flies") utilized by Ali-Scout during trip start and end points. This feature may have led to a less favorable evaluation of this type of ATIS. A second factor that may be related to the less positive findings for Ali-Scout is that this dynamic system did not seem to lead to trips that were faster or less congested, as would be expected. As mentioned earlier, this lack of a dynamic 
route guidance benefit may have been attributable to a lack of less-congested routes, or it may have resulted from an inability of the AliScout system to adequately predict traffic congestion. The Ali-Scout system predicted only recurrent traffic congestion. The dynamic information used by Ali-Scout was based on actual travel times of Ali-Scout-equipped vehicles traveling in the network (probe vehicles) averaged with estimated travel times calculated using speed limits and road classification. As such, the dynamic information for calculating a route is based partially on probe-vehicle data from that same time of day one week earlier. In this sense, there is no real-time traffic information, and nonrecurring congestion could not be identified. It may be that nonrecurring congestion information is the type of traffic information that is most important in a dynamic ATIS.

One important finding in this study is that the written instructions performed well on the measures in this study relative to the exceedingly more expensive advanced technology systems. It is important to keep in mind, however, that the written instructions condition in this study was optimal and unlike the written instructions people might receive from, for example, a friend or a person on the street. In this study, the written instructions were composed by the investigators ("experts"), neatly and clearly written, and presented to the drivers without them having to write anything. In addition, advanced technologies might provide other benefits over written instructions that were not investigated here. For example, a user of advanced navigation assistance technology would not need to ask other people for, and trust them to provide, accurate instructions. This might be a particular benefit in an unfamiliar area. Advanced technologies can also provide many features unavailable in written instructions, such as options for customizing routing (e.g., avoiding freeways or traffic control devices) and travel information (such as location of restaurants or gas stations).

Nevertheless, despite the quality of the written instructions in the present experiment and the other untested potential benefits of ATIS, the results show that advanced technologies for navigation assistance need further research and development. The present results and other studies of these ATISs (e.g., Eby, 1997; Eby, Kostyniuk, Streff, et al., 1997; Kostyniuk et al., 1997) show that people do not like and have difficulty using the "as the crow flies" distance and direction navigation information (i.e., autonomous mode for AliScout). Future systems should either avoid this information or not rely solely on it for providing navigation assistance during any part of a trip.

The same studies have found that the beaconbased infrastructure used by Ali-Scout for vehicle location and communication was problematic. Drivers occasionally lost guidance because of communication failure at a beacon, and navigation assistance was limited to the area in which the beacons were installed. This suggests that an infrastructure in which communication cannot be disrupted by roadway obstacles and that has broad coverage of an area would be preferable. One such infrastructure would be the cellular phone network already installed in many parts of the world coupled with a GPS for determining vehicle location. Finally, methods need to be devised to more accurately, and in a more timely manner, include traffic information in routing. As noted in this study, the nonrecurring traffic incidents are the most difficult to detect and include in routing algorithms, but information about them may be the most important type of traffic information for both efficient routing and user satisfaction.

\section{ACKNOWLEDGMENTS}

This work was sponsored by the Road Commission of Oakland County and the Federal Highway Administration as part of the FASTTRAC Intelligent Transportation System demonstration project. We thank Carl Christoff, Peter Emery, Sungyong In, Michelle Olk, and Claire Sheldon for their help in data collection. For numerous thoughtful discussions on this project we thank Michelle Olk, Lisa Molnar, Fredrick Streff, Steven Underwood, and Richard Wallace. Hans Joksch provided valuable assistance on the data analyses. We thank Deborah A. Mitta and two anonymous reviewers for their comments on earlier drafts of this 
paper. A preliminary report of this work was presented at the Fourth World Congress on Intelligent Transportation Systems in Berlin, Germany (Eby \& Kostyniuk, 1997).

\section{REFERENCES}

Chu, X. (1994). The effects of age on the driving habits of the elderly: Evidence from the 1990 NPTS (Report No. NUTI93USF3.2). Tampa, FL: National Urban Transit Institute.

Eby, D. W. (1997). How people use in-vehicle navigation assistance systems. In Proceedings of the 2nd Annual Land Navigation Conference (pp. 311-319) Ann Arbor, MI: Environmental Research Institute of Michigan.

Eby, D. W., \& Kostyniuk, L. P. (1997). An on-the-road comparison of in-vehicle navigation assistance technologies: The FASTTRAC troika study. In Proceedings of the 4th World Congress on Intelligent Transportation Systems, Berlin, Germany [CDROM]. Brussels, Belgium: ITS Congress Association.

Eby, D. W., \& Kostyniuk, L. P. (1998). Maintaining older driver mobility and well-being with traveler information systems. Transportation Quarterly, 52, 45-53.

Eby, D. W., Kostyniuk, L. P., Christoff, C., Hopp, M. L., \& Streff, F. M. (1997). An on-the-road comparison of in-vehicle navigation assistance systems: The FAST-TRAC troika study (Report No. UMTRI-97-05). Ann Arbor, MI: University of Michigan Transportation Research Institute.

Eby, D. W., Kostyniuk, L. P., Streff, F. M., \& Hopp, M. L. (1997). Evaluating the perceptions and behaviors of Ali-Scout users in a naturalistic setting (Report No. UMTRI-97-08). Ann Arbor, MI: University of Michigan Transportation Research Institute.

Eby, D. W., Trombley, D. A., Molnar, L. J., \& Shope, J. T. (1998). The assessment of older capabilities: A review of the literature (Report No. UMTRI-98-24). Ann Arbor, MI: University of Michigan Transportation Research Institute.

Fairclough, S. H., Ashby, M. C., Ross, T., \& Parkes, A. M. (1991). Effects of handsfree telephone use on driving behaviour. In International Symposium on Automotive Technology and Automation, 24th Proceedings of the Dedicated Conference on Road Transport Informatics (RTI)/Intelligent Vehicle Highway Systems (IVHS) (pp. 403-409). Croyden, UK: Automotive Automation.

Fastenmeier, W., Haller, R., \& Lerner, G. (1994). A preliminary safety evaluation of route guidance comparing different MMI concepts. In Proceedings of the 1st World Congress on Applications of Transport Telematics and Intelligent VehicleHighway Systems (pp. 1750-1757). Boston: Artech House.

Inman, V., Sanchez, R., Porter, C., \& Bernstein, L. (1995). TravTek evaluation: Yoked driver study. (Report No. FHWA-RD-94139). Washington, DC: Federal Highway Administration.

IVHS America. (1992). Strategic plan for intelligent vehicle-highway systems (IVHS) in the United States (final draft). Washington, DC: Author.

Kantowitz, S. C., Kantowitz, B. H., \& Hanowski, R. J. (1995). The Battelle route guidance simulator: A low-cost tool for studying driver response to advanced navigation systems. In
Proceedings of the 1995 Vehicle Navigation and Information Systems Conference (pp. 104-109). Piscataway, NJ: IEEE.

Kostyniuk, L. P., Eby, D. W., Christoff, C., Hopp, M. L., \& Streff, F. M. (1997). The FAST-TRAC natural use leased-car study: An evaluation of user perceptions and behaviors of Ali-Scout by age and gender (Report No. UMTRI-97-09). Ann Arbor, MI: University of Michigan Transportation Research Institute.

Mehta, C. R., \& Patel, N. R. (1983). A network algorithm for performing Fisher's Exact Test in rXc contingency tables. Journal of the American Statistical Association, 78, 427-434.

Miller, G. A. (1956). The magical number seven plus or minus two: Some limits on our capacity for processing information. Psychological Review, 63, 81-97.

Molnar, L. J., Eby, D. W., \& Hopp, M. L. (1996). Developing information systems for the driving tourist: A literature review (Report No. UMTRI-96-11). Ann Arbor, MI: University of Michigan Transportation Research Institute

Parkes, A. M., Ashby, M. C., \& Fairclough, S. H. (1991). The effects of different in-vehicle route information displays on driver behavior. In Proceedings of the 1991 Vehicle Navigation \& Information Systems Conference (pp. 61-70). Warrendale, PA: Society of Automotive Engineers.

Schofer, J. L., Koppelman, F. S., Webster, R. G., Berka, S., \& Peng, T. (1996). Field test of the effectiveness of ADVANCE dynamic route guidance on a suburban arterial street network (ADVANCE Project Document No. 8463.01). Evanston, IL: Northwestern University Transportation Center.

Smiley, A., Vernet, M., Labiale, G., \& Pauzié, A. (1994). Navigation guidance displays: Impact on driver performance. In Proceedings of the 1st World Congress on Applications of Transport Telematics and Intelligent Vehicle-Highway Systems (pp. 1852-1859). Boston: Artech House.

Srinivasan, R., Landau, F. H., \& Jovanis, P. P. (1995). A simulator evaluation of five in-vehicle route guidance systems. In Proceedings of the 1995 Vehicle Navigation and Information Systems Conference (pp. 90-95). Piscataway, NJ: IEEE.

Walker, J., Alicandri, E., Sedney, C., \& Roberts, K. (1990). Invehicle navigation devices: Effects on the safety of driver performance (Report No. FHWA-RD-90-053). Washington, DC: Federal Highway Administration.

David W. Eby is an assistant research scientist at the University of Michigan Transportation Research Institute, Social and Behavioral Analysis Division. $\mathrm{He}$ received his Ph.D. in psychology from the University of California, Santa Barbara, in 1991.

Lidia P. Kostyniuk is an associate research scientist at the University of Michigan Transportation Research Institute, Social and Behavioral Analysis Division. She received her Ph.D. in civil engineering from the State University of New York at Buffalo in 1975.

Date received: July 29, 1997

Date accepted: October 22, 1998 